\title{
Experimental verification of Acuros XB in the presence of lung-equivalent heterogeneities
}

\author{
M. Bueno, M. A. Duch \\ Institut de Tècniques Energètiques, Universitat Politècnica de Catalunya, 08028 \\ Barcelona,Spain \\ D. Jurado-Bruggeman, S. Agramunt-Chaler and C. Muñoz-Montplet \\ Servei de Física Mèdica i Protecció Radiològica, Institut Català d'Oncologia-Girona, \\ 17007 Girona, Spain
}

\begin{abstract}
Acuros $X B(A X B)$ is a deterministic algorithm that directly accounts for the effects of heterogeneities and improves the accuracy of photon dose calculations in radiotherapy. Nevertheless, the accuracy of AXB inside lung has not been yet fully examined by means of experimental measurements. The aim of this study was to evaluate the accuracy of $A X B$ in the presence of lung using ${ }^{7} \mathrm{Li}$-based thermoluminescent dosimeters (TLD-700). Percentage depth-dose distributions (PDD) obtained by $\mathrm{AXB}$ on a slab water-equivalent phantom with a lungequivalent heterogeneity were compared with the TLD-700 measurements for 6 MV and $18 \mathrm{MV}$ photon beams and a set of field sizes ranging from $2 \times 2 \mathrm{~cm}^{2}$ to $20 \times 20 \mathrm{~cm}^{2}$. Dose distributions obtained by the Anisotropic Analytical Algorithm (AAA) were also included in the comparison as a reference to a nondeterministic dose calculation algorithm. The agreement between AXB and the TLD results was kept within clinical tolerance levels $(3 \%)$ for all beam configurations. On the contrary, AAA failed at correctly predicting the absorbed dose when the lateral electronic equilibrium was lost. $A X B$ is capable of providing reliable dose estimations in the presence of lung and clearly outperforms AAA.
\end{abstract}

Keywords: Acuros XB, TLD, heterogeneities, lung

\section{Introduction}

Li-based thermoluminescent detectors (TLDs) are suitable for the experimental verification of dose calculation algorithms in radiotherapy [1, 2]. The thermoluminescent material is tissue-equivalent, which makes it especially use-

5 ful for validation inside lung where ionization chambers (IC) cannot generally

\footnotetext{
${ }^{*}$ Corresponding author

Email address: mbuenovizcarrågmail l.com (M. Bueno, M. A. Duch)
} 
be used[3]. The presence of such low-density heterogeneities constitute a challenge to most correction-based and convolution-superposition dose calculation algorithms, which are usually unable to accurately determine the absorbed dose in these conditions $[4,5]$.

10 In 2010, the Acuros XB (AXB, Varian Medical Systems, Palo Alto, CA), was presented as a novel deterministic dose calculation algorithm that directly accounts for the effects of heterogeneities in patient dose calculations by explicitly solving the linear Boltzmann transport equation [6]. Therefore, AXB can converge to the same solution as Monte Carlo (MC) simulations even in

15 lung [7], which undoubtedly represents a significant improvement over the nondeterministic dose calculation algorithms.

Over the past years, few studies have been devoted to experimentally evaluate the accuracy of $A X B$ in lung despite it is of utmost interest considering that both $A X B$ and $M C$ calculations are affected by the same sources of uncertainty.

${ }_{20}$ Among them, most investigations have performed measurements at or near the heterogeneity interface and just a couple have done it inside the lung-equivalent material $[8,9]$. In these investigations, a small number of points per plan have been evaluated and the reported uncertainties sometimes exceeded the clinical tolerance level that is commonly accepted (3\%) [10].

25 In this study, the accuracy of the AXB dose calculation algorithm in a slab water phantom with a lung-equivalent heterogeneity was thoroughly examined by means of TLDs. The behavior of AXB was compared to that of the Anisotropical Analytical Algorithm (AAA) as a reference of a non-deterministic algorithm.

\section{Methods and materials}

Two photon beam energies, 6 and $18 \mathrm{MV}$, from a Varian Clinac $2100 \mathrm{C} / \mathrm{D}$ (Varian, Palo Alto, CA) linear accelerator were considered. Percentage depthdose (PDD) curves for field sizes ranging from $2 \times 2 \mathrm{~cm}$ to $20 \times 20 \mathrm{~cm}^{2}$ were evaluated on a water-equivalent slab phantom (PTW RW3, electron density

35 relative to water $\rho_{\mathrm{e}}^{\mathrm{w}}=1.012$ ) with a lung-equivalent heterogeneity (Computerized Imaging Reference Systems (CIRS), Norfolk (VA), $\left.p^{\mathrm{w}}=0.195\right)$. The studied configuration was $5 \mathrm{~cm}$ of RW3 followed by $13 \mathrm{~cm}$ of lung and then by $10 \mathrm{~cm}$ of RW3. It was built using $30 \times 30 \mathrm{~cm}^{2}$ slabs with thicknesses ranging from 0.1 to $5 \mathrm{~cm}$.

\section{2.1. $A X B$ calculations}

Dose distributions were obtained with the $A X B$ algorithm (v. 13). and with the AAA (v. 10.28.0). A virtual phantom was generated from the CT image (Optima CT580RT CT scanner, General Electric Healthcare, Fairfield, CT) of the experimental configuration. The field-of-view was set to $65 \mathrm{~cm}$ and the voxel

45 size was fixed to $1.27 \times 1.27 \times 5 \mathrm{~mm}^{3}$. The material assigned to each region of the phantom (water or lung) was selected among the biological materials available in the Eclipse ${ }^{\mathrm{TM}}$ treatment planning system. 
The three source model used in $A X B$ and $A A A$ was automatically configured using the default focal spot size of $1 \mathrm{~mm}$ in $\mathrm{x}$ and $\mathrm{y}$ directions [11].

50

\subsection{Experimental measurements}

Dose measurements within the water-like regions of the phantom were carried out by ICs, as they are the detectors of reference in water.(author?) [3] A $0.016 \mathrm{~cm}^{3}$ PinPoint 31016 (PTW, Freiburg, Germany) was used to determine the absorbed dose for the smallest field sizes $\left(2 \times 2 \mathrm{~cm}^{2}\right.$ and $\left.5 \times 5 \mathrm{~cm}^{2}\right)$ and a ${ }_{55} 0.35 \mathrm{~cm}^{3}$ Roos (PTW, Freiburg, Germany) was used for the largest field sizes $\left(10 \times 10 \mathrm{~cm}^{2}\right.$ and $\left.20 \times 20 \mathrm{~cm}^{2}\right)$. Both were connected to the UNIDOS electrometer (PTW, Freiburg, Germany).

No IC measurements were carried out within the lung heterogeneity because perturbation correction factors for these ICs are unknown in media other that water. Instead, TLDs were preferred to measure the dose in lung.

The TLDs used in this study were ${ }^{7} \mathrm{LiF}: \mathrm{Mg}, \mathrm{Ti}$ chips type TLD-700 (Thermo Fisher Scientific Inc., Erlangen, Germany). ${ }^{7} \mathrm{Li}$ compounds were chosen to avoid the interference of neutron fields for $18 \mathrm{MV}$ photons generated by linear accelerators. TLD-700 are squared pellets of $3.1 \times 3.1 \mathrm{~mm}^{2}$ and $0.9 \mathrm{~mm}$ thickness. The thermoluminescent material of TLD700 has an effective atomic number similar to soft tissue, which makes it suitable for absorbed dose measurements in lung. The suitability of this detector to provide reliable dose estimations has been reported elsewhere in similar conditions [5].

Characterization of TLD-700 can be found in the literature [12]. TLD-700

70 was found to fulfill the requirements of (i) flat energy response for the photon energies involved in this study, (ii) linear response with dose within the dose range handled herein, and (iii) reproducibility of results.

Before each irradiation, standard annealing was carried out in a PTW-TLDO oven. The annealing cycle consisted in $1 \mathrm{~h}$ at $400^{\circ} \mathrm{C}$ followed by $2 \mathrm{~h}$ at $100^{\circ} \mathrm{C}$.

75 Readout was carried out with a Thermo Scientific Harshaw 5500 hot gas reader. The readout procedure consisted of a pre-heating phase at $135^{\circ} \mathrm{C}$ during $10 \mathrm{~s}$ followed by a reading phase in which detectors are heated up to $270^{\circ} \mathrm{C}$ at a linear rate of $25^{\circ} \mathrm{C} \cdot \mathrm{s}^{-1}$ during approximately $10 \mathrm{~s}$.

To improve the dosimeters accuracy, individual calibration factors were es-

so tablished for each detector, and stability checks were performed periodically with a caesium-137 beam, as detailed by Ginjaume et al. [12].

Aiming at reducing the statistical uncertainty of results, up to 9 detectors (limited by the field size) were used at each depth and measurements were repeated up to three times.

\section{Results}

Calculated and measured PDDs are shown in figure 1 for $6 \mathrm{MV}$ and $18 \mathrm{MV}$ photon beams (left and right columns, respectively). Percentage differences between the $A X B$ relative dose predictions and the experimental results computed as $D^{A X B}(\%)-D^{T L D}, I C(\%)$ are depicted in the insert of the figures. 
TLD-700 relative dose estimations were obtained within $\pm 1-2 \%$ statistical uncertainty in $98 \%$ of the cases. Error bars in figure 1 correspond to the associated standard deviations (1 SD). Statistical uncertainties associated to the IC measurements were kept around $1 \%$ in all cases. Error bars are not shown in figure 1 for clarity.

AXB dose distributions agreed within $1.5 \%$ with the IC measurements for the two beam energies and all considered field sizes. The agreement with respect to the TLD-700 measurements was generally kept below $2 \%$ at all depths both in the water-like regions and within the heterogeneity. Nevertheless, more noticeable discrepancies were registered within the heterogeneity for the $2 \times 2 \mathrm{~cm}^{2}$ field size, especially for the $18 \mathrm{MV}$ photon beam (see figures $1 \mathrm{a}$ and $1 \mathrm{~b}$ ). For this field size, average deviations within the lung-equivalent material were $-1.16 \%$ and $2.6 \%$ for the $6 \mathrm{MV}$ and $18 \mathrm{MV}$ photon beams, respectively. Maximum point differences of $-3.1 \%$ and $-4.2 \%$ were registered for the lowest and the highest considered beam energy, respectively.

As shown in figure 2, AAA dose estimations agreed within 2-3\% with AXB and the experimental measurements along the heterogeneous phantom after the build-up region with a couple of exceptions. First, significant discrepancies were observed within the lung-equivalent material for the smallest field size, for which AAA overestimated the absorbed dose with respect to AXB by up to $4.4 \%$ and $19.6 \%$, for $6 \mathrm{MV}$ and $18 \mathrm{MV}$, respectively. Second, differences up to $5.1 \%$ were registered within the water-equivalent region after the heterogeneity for the 6 MV photon beam, especially for the two largest field sizes upon study.

\section{Discussion}

The accuracy provided by AXB (v. 13) in predicting the absorbed dose within a water phantom with a lung-equivalent heterogeneity was examined by means of ionization chambers (IC) and $7 \mathrm{Li}$-based thermoluminescent detectors (TLD-700). Excellent agreement was found between AXB and the experimental measurements within the water regions of the heterogeneous phantom. Discrepancies between the AXB and the TLD-700 relative dose estimations in lung were generally kept within the accepted clinical tolerance of $2 \%$ [10] in all considered cases. Nevertheless, it should be noticed that larger deviations were registered within the lung-equivalent heterogeneity for the $2 \times 2 \mathrm{~cm}^{2}$ field size, especially for the $18 \mathrm{MV}$ photon beam energy. This beam configuration is of particular interest because the lack of lateral electronic equilibrium in lung is very significant. Under these conditions, the measurement of dose to water becomes difficult [13]. The differing density of the detector and the medium exacerbates the effects of electronic disequilibrium by compensating for it (density of the TLD-700 is greater than density of the lung-equivalent material), leading to dose over-read [14]. This phenomenon might be minimized by considering thinner detection volumes in the radiation beam direction. Therefore, ultra-thin TLDs should be preferred over standard-thickness TLDs for experimental verification of small fields inside low-density heterogeneities to ensure maximum accuracy. 


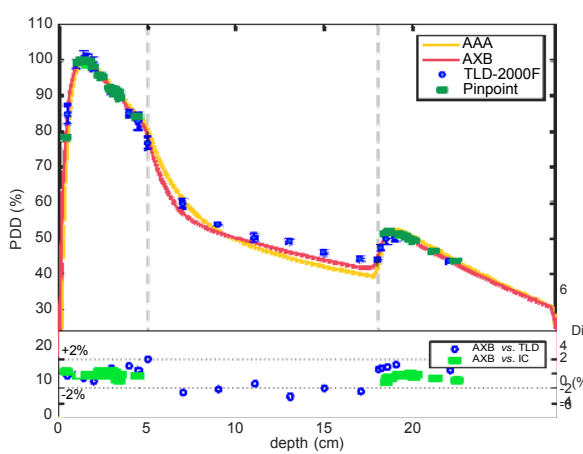

(a) $6 \mathrm{MV}-2 \times 2 \mathrm{~cm}^{2}$

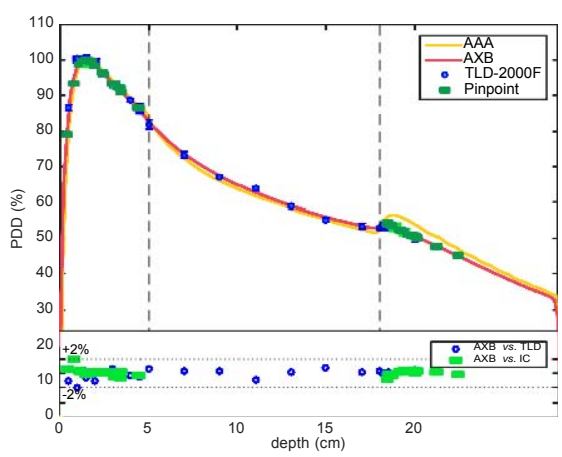

(c) $6 \mathrm{MV}-5 \times 5 \mathrm{~cm}^{2}$

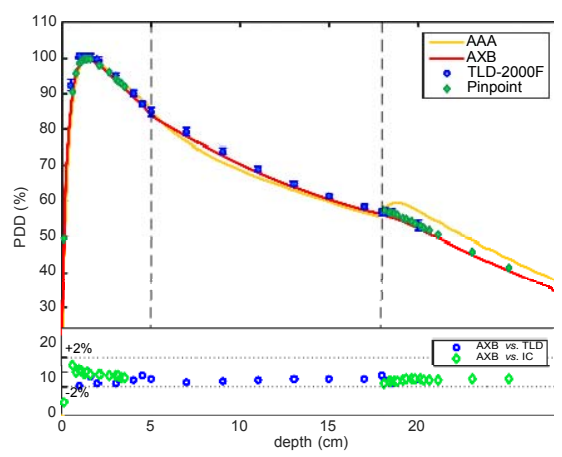

(e) $6 \mathrm{MV}-10 \times 10 \mathrm{~cm}^{2}$

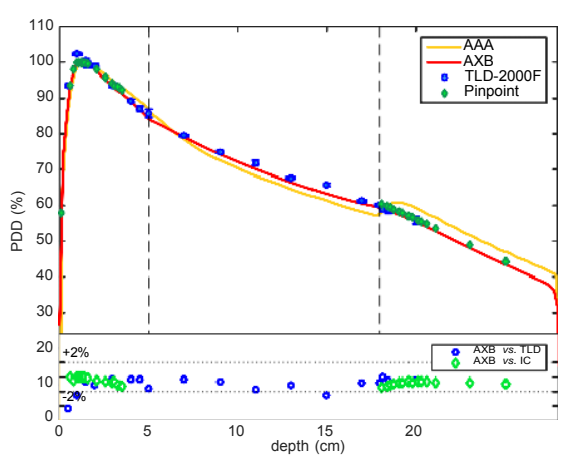

(g) $6 \mathrm{MV}-20 \times 20 \mathrm{~cm}^{2}$

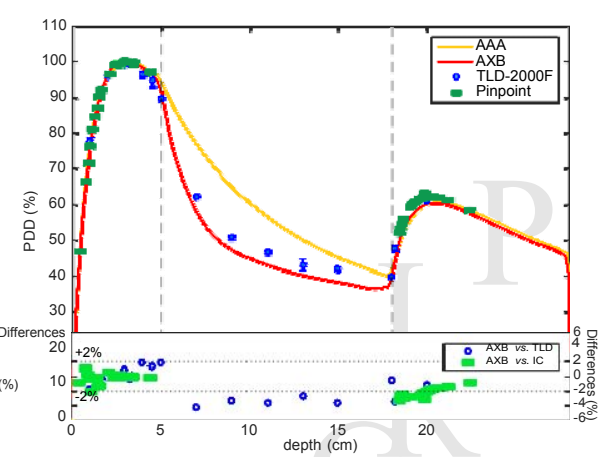

(b) $18 \mathrm{MV}-2 \times 2 \mathrm{~cm}^{2}$

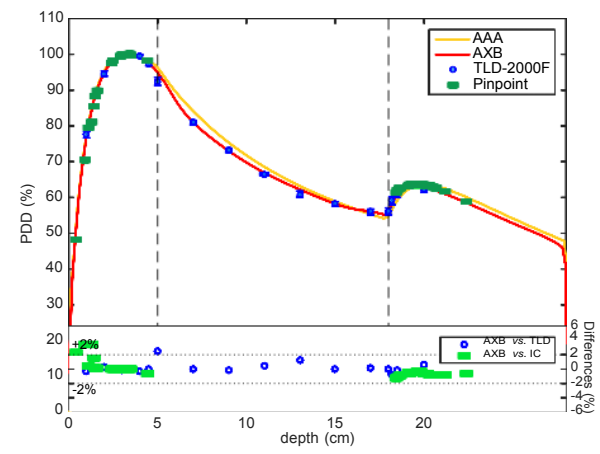

(d) $18 \mathrm{MV}-5 \times 5 \mathrm{~cm}^{2}$

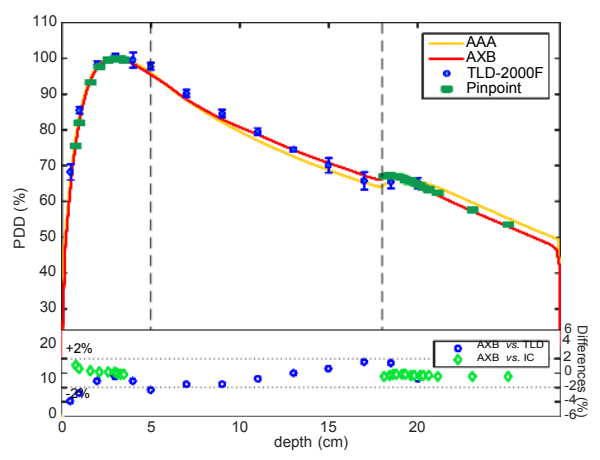

(f) $18 \mathrm{MV}-10 \times 10 \mathrm{~cm}^{2}$

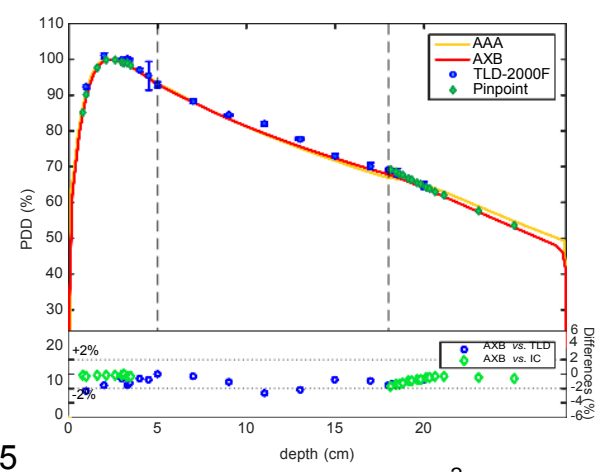

(h) $18 \mathrm{MV}-20 \times 20 \mathrm{~cm}^{2}$

Figure 1: PDD curves for $6 \mathrm{MV}$ (left column) and $18 \mathrm{MV}$ (right column) photon beams and different field sizes (rows) determined by AXB, AAA, and the experimental measurements. Percentage differences are depicted in the inserts. 


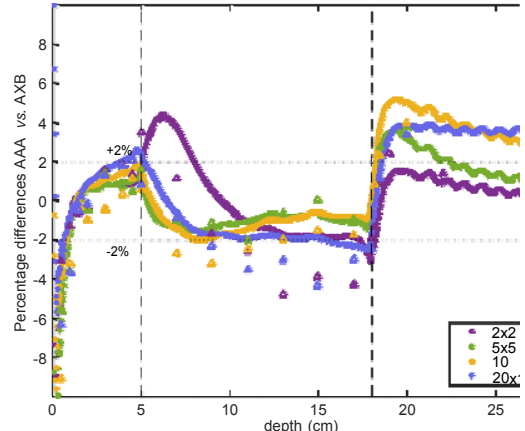

(a) $6 \mathrm{MV}$

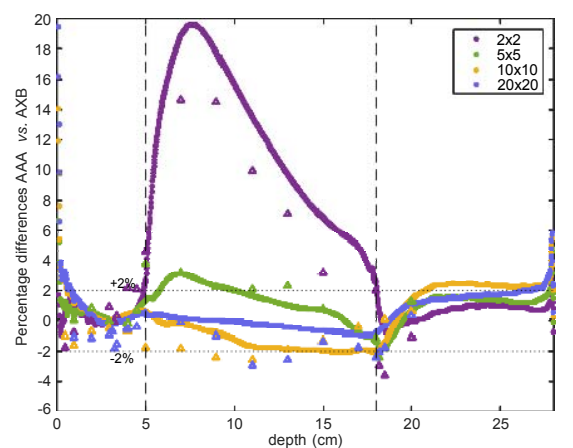

(b) $18 \mathrm{MV}$

Figure 2: Percentage differences between AAA and AXB for $6 \mathrm{MV}$ (left) and $18 \mathrm{MV}$ (right) photon beams and different field sizes. Discrepancies of AAA with respect to TLD are also included (small triangles for all field sizes, consistently colored).

AAA provided reasonably accurate dose estimations within the lung-equivalent material in all cases except for the smallest field and highest beam energy considered. AAA was unable to properly handle the electronic disequilibrium in lung and could not correctly predict the absorbed dose within the heterogeneity, which is in good agreement with what has been already reported in the literature $[4,15]$. Furthermore, AAA fails at providing accurate dose estimations within the water region after the heterogeneity, as other convolution-based algorithms have been reported to do [16].

\section{Conclusions}

AXB (v. 13) provides accurate dose estimations in the presence of lung heterogeneities for high energy photon beams and represents a significant improvement over standard convolution-superposition algorithms, such as AAA, for dose calculations in radiotherapy.

\section{Acknowledgments}

This work has been partially funded by the Spanish Ministry of Science and Innovation (MICINN) and the Regional European Development Funds (FEDER) under contract UNPC10-4E-913.

\section{References}

[1] T. Han, D. Mourtada, K. Kisling, J. K. Mikell, D. Followill, R. Howell, Experimental validation of deterministic Acuros XB algorithm for IMRT and VMAT dose calculations with the Radiological Physics Center?s head and neck phantom, Med. Phys. 39 (2012) 2193-2202. 
[2] M. W. K. Kan, L. H. T. Leung, R. W. K. So, P. K. N. Yu, Experimental verification of the Acuros $\mathrm{XB}$ and $\mathrm{AAA}$ dose calculation adjacent to heterogeneous media for IMRT and RapidArc of nasopharygeal carcinoma, Med. Phys. 40 (3) (2013) 031714.

[10] Determination of absorbed dose in a patient irradiated by beams of $X$ or gamma rays in radiotherapy procedures, Tech. Rep. 24, International Commission on Radiation Units and Measurements, Bethesda, MD (1976).

[11] L. Tillikainen, S. Siljamäli, H. Helminen, J. Alakuijala, J. Pyyry, Determination of parameters for multiple-source model of megavoltage photon beams using optimization methods, Phys. Med. Biol. 52 (2007) 1441-1467.

[12] M. Ginjaume, X. Ortega, M. Duch, N. Jornet, A. Snchez-Reyes, Characteristics of lif:mg,cu,p for clinical applications, Radiat. Meas. 85 (1999) 389-391. 
195 [13] I. Das, G. Ding, A. Ahnesjö, Small fields: Nonequilibrium radiation dosimetry, Med. Phys. 35 (2008) 206-215.

[14] A. J. Scott, S. Kumar, A. E. Nahum, J. Fenwick, Characterizing the influence of detector density on dosimeter response in non-equilibrium small photon fields, Phys. Med. Biol. 57 (2012) 4461-4476.

[15] T. Han, J. K. Mikell, M. Salehpour, F. Mourtada, Dosimetric comparison of Acuros XB deterministic radiation transport method with Monte Carlo and model-based convolution methods in heterogeneous media, Med. Phys. 38 (2011) 2651-2664.

[16] P. Carrasco, N. Jornet, M. A. Duch, L. Weber, M. Ginjaume, T. Eudaldo,

D. Jurado, A. Ruiz, M. Ribas, Comparison of dose calculation algorithms in phantoms with lung equivalent heterogeneities under conditions of lateral electronic disequilibrium, Med. Phys. 31 (2004) 2899-2911. 
Highlights on the experimental verification of Acuros XB in the presence of lungequivalent heterogeneities

- TLD-700 are used to validate the accuracy of Acuros XB inside lung-equivalent media.

- Acuros XB (AXB) provides accurate dose distributions for a wide range of field sizes.

- AXB represents a significant improvement over standard nondeterministic algorithms. 